\title{
Game-Based Learning in Technology Management Education: A Novel Business Simulation
}

\author{
doi:10.3991/ijet.v6i1.1522 \\ M. Günther ${ }^{1}$, E. Kiesling ${ }^{2}$ and C. Stummer ${ }^{1}$ \\ ${ }^{1}$ Bielefeld University, Bielefeld, Germany \\ ${ }^{2}$ University of Vienna, Vienna, Austria
}

\begin{abstract}
Management games allow students to obtain valuable first-hand experience that is of particular value in a field such as technology management, which usually involves substantial risk as well as significant time lags between a managerial decision and its effects. In drawing from more than fifteen years of experience with management games in higher education, we have developed a management simulation for teaching technology management, a field that is increasingly regarded an important area of study for engineering students. This paper outlines a blended-learning design for a course on technology management, describes the various didactical elements as well as their effects in class, and addresses the technical implementation of the management game as a service-oriented multitier application in Java.
\end{abstract}

Index Terms-experiential learning; management game; simulation; technology management.

\section{INTRODUCTION}

Technological innovations are essential for the success and survival of firms in today's dynamic market place. It is therefore not surprising that managers devote large amounts of resources to research and the development of new technologies. At the same time, uncertainty regarding the success of technological endeavors is typically high. Given its strategic importance, the resources at stake, and the substantial risks involved, a systematic approach to the management of technology is considered indispensable by both the scientific community and practitioners.

Consequently, technology management has become a highly relevant area of study - not only in most business schools [1], but also in engineering management education, that deals extensively with technology management issues as means to bridge the gap between the fields of engineering and business (for examples in the US cf. [2]). This is also true for vertically-focused engineering curricula, which are however giving way to integrated programs encompassing multiple disciplines [3]. Accordingly, an increasing number of engineering programs integrate technology management in their curricula, thereby strengthening the trend towards broader scholastic experiences by offering engineering students an interdisciplinary perspective.

In practice, corresponding technology management courses more often than not are taught in a "traditional" lecture format that may be complemented by case analy- ses [4]. Management gaming (also commonly termed "management simulations", "management (flight) simulators" or "business gaming simulations"; for an overview on the history and a discussion of the differences in terminology cf. [5]) have been applied successfully in many areas of management education for more than 40 years [68]. However, they do not appear to be used prevalently in technology management education.

As means for experiential learning [9], management games allow participants to assume the role of decisionmakers that compete against each other in a "safe" artificial environment. By shortening real-world decision cycles from years or decades to hours or minutes in the simulation, the learning process can be accelerated dramatically, which should help participants to gain a better understanding of the real-life situation the simulation game refers to. The main objective of using management games as tools to enhance learning is to give participants the opportunity to apply theoretical knowledge in close-toreality situations in order to develop decision-making competence, rather than solely acquiring theoretical knowledge. In a well-designed and implemented simulation, this may lead to the synthesis of theory and practice [5]. Management simulations can thus be seen as an appropriate response to the criticisms against the "knowledge acquisition approach" (cf. [10]) in management education. They are especially useful in fields like technology management, where delays, the large amounts of resources at stake, and the high risk involved prohibit reallife experimentation.

The remainder of this paper is organized as follows: Section 2 delineates the conceptualization of the game and discusses thematic aspects covered in the simulation. Section 3 outlines how the management simulation is embedded in an overall didactic design. Section 4 provides details about the implementation as a service-oriented multitier application in Java. The paper concludes in Section 5 with a summary and an outlook on further research.

\section{CONCEPTUALIZATION}

In developing a management simulation for Masterlevel students, we could draw upon more than fifteen years of experience with management games in higher education, which allowed us to "cherry-pick" the most effective didactical means in order to tweak the learning outcomes. To account for the integral nature of decisions in the field of technology management, the simulation also comprises elements from other areas such as marketing, 
production, and finance, and may thus be considered a total management simulation. The main emphasis, however, is on the strategic planning of research and development (R\&D) investment, the adoption of and migration to new technologies, and the management of patents and licensing. The simulation is generic in that it does not represent any specific industry, but can be tailored through parameterization using an instructor tool. The tool also allows for the creation of scenarios with varying degrees of complexity through optional features, such as stockkeeping, cooperative research or patents and licensing. Furthermore, the instructor may define all functions by setting control points and selecting linear, polynomial or cubic spline interpolation.

In each game, a single participant or a group of participants takes control of a virtual firm. At the beginning, all firms are homogeneous in that they possess the same amount of initial capital, the same amount of knowledge and an equal number as well as equal kinds of products and production facilities. Each game follows an iterative process in which participants are supplied with various reports on the performance of their firm that they may use to evaluate their strategy and possibly adapt it before submitting their decisions for the next period. As soon as all decisions for a period have been received, the results are processed automatically and financial statements and additional reports are generated before the game proceeds to the next round. This procedure is repeated until some terminating condition, such as a pre-determined time or a number of periods, is met or until the instructor ends the game manually.

Firms compete in a simulated market by developing and launching products. Before doing so, they need to conduct (basic) research to accumulate knowledge, which eventually leads to the "discovery" of advanced technologies. When such a new technology becomes available to a firm, it may be adopted and serve as a basis for subsequent product development. The resulting products are characterized by a number of attributes which are defined by the instructor, who may choose generic measures such as functionality, performance, usability, durability etc., or scenario-specific measures relevant in the simulated industry (e.g. engine performance, safety or handling in the automotive industry). Strategic decisions regarding resource allocation are a key element of the simulation. Participants need to weigh investments in research (which may lead to breakthroughs and performance leaps in the long run) against investments in the development of more sophisticated products based on current technology and/or against investments in process development (which reduces production costs). Once a technology becomes available, participants need to determine a plan for investing in the new technology and for phasing out existing technologies. Thus, participants may test various strategies, such as early adopter or fast follower strategies, and experience their respective advantages and drawbacks.

As soon as a satisfying performance increase has been achieved in a technology, firms may decide to launch new products at the market where they compete for market share with existing products that consumers are already familiar with. In our management game, we used an agent-based approach for simulating the diffusion of information about new technologies and products through the social network of consumers. To generate realistic diffusion patterns and simulate buying behavior observed in the real world, the instructor may divide the market into several heterogeneous segments with distinct characteristics regarding propensity to innovate, preferences, price sensitivity, brand loyalty, sensitivity to advertising, and communication behavior, which determines the diffusion of information about new technologies and products in the segment. Revenue generated from product sales is used to cover costs of production, stock-keeping, business intelligence and licensing, among others. Profits may be reinvested in research, development of the various performance dimensions of technologies, process development, construction of new production facilities, or advertising.

Once a game has ended, participants receive a final report that summarizes results and provides more detailed information that is not available during the game. Overall participant performance is measured by equity held at the end of the game as well as by the amount of technological progress achieved.

\section{DIDACTIC DESIGN}

In a management game, participants interact with/within a modeled system that represents a part of reality. These interactions follow a general course (see Figure 1) where participants choose actions through decisions that are then entered - either directly (as in the online version) or via an instructor (as in the offline version) - into the simulation program. Based on these decisions and global conditions (such as market, inflation etc.) the system reacts according to the rules of the implemented model. Finally, feedback (e.g. on market development, sales etc.) is provided to the participants through various reports that, in turn, form the basis for further user action, thus restarting the loop.

Even though this general course, and therefore the management simulation itself, forms the center of the didactical frame, it has to be embedded into a broader (blended) learning concept to meet the learning objectives. To this end, the simulation game is not only played with various settings, levels of complexity and difficulty in both online and offline environments, but is also supplemented with accompanying measures and tasks. Furthermore, the management game can be embedded into a broader course design, as (engineering) students need to possess at least basic knowledge about technology management before engaging in the game. At the University of Vienna's Faculty of Computer Science, for example, the management simulation is integrated into a larger course that commences by imparting basic theory, provides students contact with business practitioners by means of excursions and invited talks, and also requires

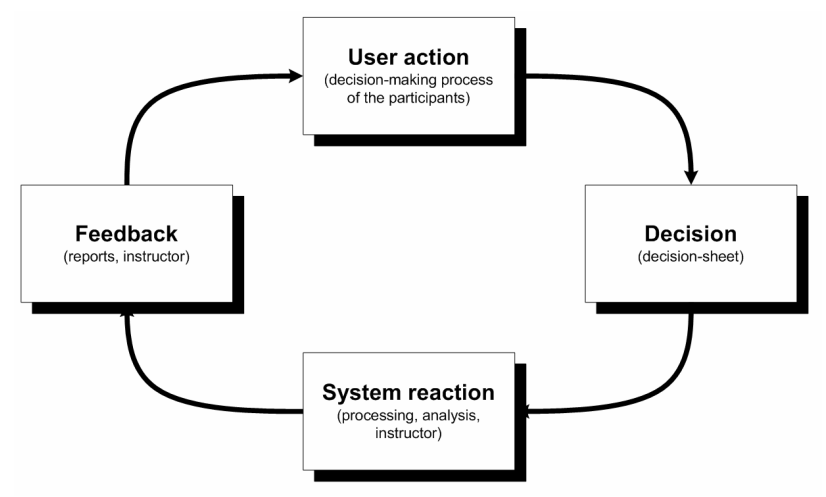

Figure 1. General course of a management game. 


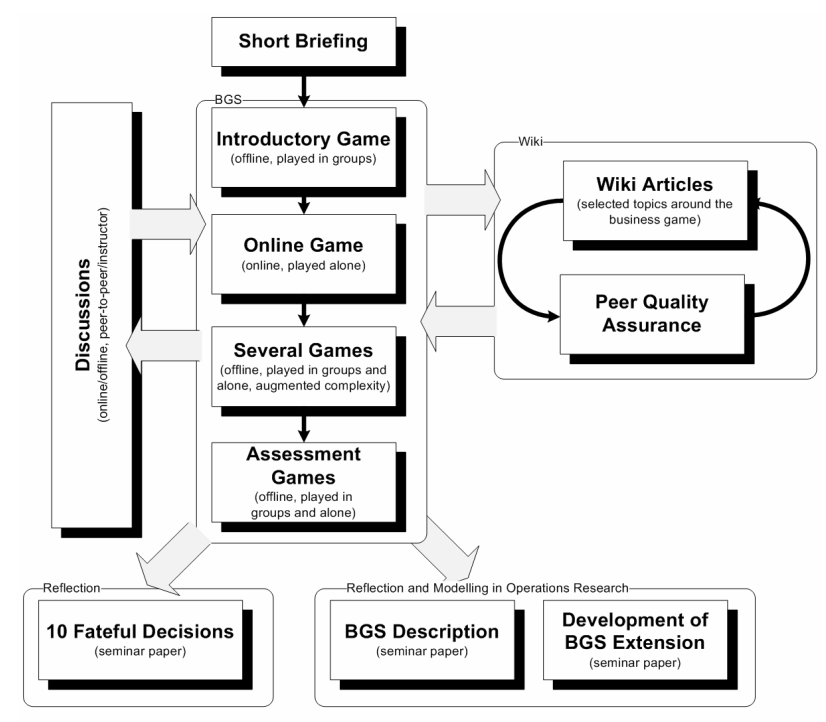

Figure 2. Overview of the didactic concept of the management game.

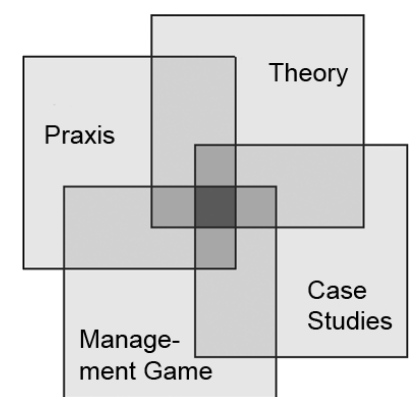

Figure 3. Integration of the management game in a course design.

the preparation and discussion of case studies on technology management in class (see Figure 3). Thus, participants are well prepared when they start to play the gaming simulation.

The didactic concept of the management game is illustrated in Figure 2. Before the first game is started, participants are briefed on the overall setting as well as on technicalities concerning the decisions they need to make. However, no details about the inner workings of the game are revealed, since the participants should learn by doing. Next, a first (offline) introductory game is played in class, which bears several advantages. First, the instructor may directly react to questions without the typical time lag that occurs in an online setting. Second, the participants' decision-making process may be observed, which helps to spot problems or mistakes. Also the atmosphere within the group can be monitored more easily in a classroom setting, which helps to prevent frustration as a consequence of unexpected results. Even though direct assistance might be one possibility, following the learning-by-doing approach the instructor should concentrate on the stimulation and moderation of the discussion between participants. Moreover, students can, should and do help each other when performing tasks like calculating a "good" price, analyzing data, drawing graphs etc. To encourage peer-topeer interaction during the first game, each firm is managed by two students at the beginning. As the participants should focus on the technology management aspects instead of the intricacies of a web-interface, the decisions are submitted on simple paper forms at this stage. The instructor enters the data manually and delivers the results on paper, which provides the opportunity to directly give (additional) feedback. However, these advantages of the offline version come at the cost of an increased workload so that generally more than one instructor is needed at this stage to process the data. Finally it should be noted that the complexity of the simulation is reduced during the introductory game by disabling optional aspects such as patents or licensing.

Once the initial game has ended, the web user interface is explained thoroughly to the participants in order to avoid difficulties during the subsequent online phase. During this phase, the game is played online over a time period of six weeks with two decision rounds per week. As opposed to games played in a classroom setting, participants can analyze their strategies and prepare their decisions without any time pressure. Also, in contrast to the first game, each student is fully responsible for managing their own firm in order to prevent free-rider problems. Nevertheless, both peer-to-peer and student-to-instructor communication is crucial and thus encouraged also in the online phase by providing proper channels like web forums. Experience shows that these communication channels are primarily used for questions to the instructor, while peer-to-peer interaction hardly takes place online. However, anecdotal evidence suggests that informal information exchange between students does occur offline (e.g. in the cafeteria), but is limited to selected colleagues, which indicates that students apparently regard information asymmetry as a key strategic advantage that they try to sustain. Note that the discussion forum is currently not part of the management game itself. Instead, the communication features of a learning management system (LMS) are used, which constitutes a "media disruption".

During the online phase, participants are required to prepare papers on selected topics related to the management game in order to complement the learning-by-doing approach with more theoretical reflections. The articles are co-authored in groups using a Wiki, which is integrated in the LMS. According to our experience, collaborative online writing appears to be a new concept for most of our students, which is why a basic introduction on the use of Wikis is (still) crucial. This task allows participants not only to gain experience with a new form of collaboration, but also enables them to share with other students what they have learned about the theoretical concepts embodied in the management game. This knowledge is essential for meeting the learning objectives of the course, which obviously go beyond winning the management game. A problem we frequently encountered was that students hardly ever read the articles of other participants, which may partly be attributed to the low quality of some contributions. To alleviate this problem, we decided to introduce peer quality assurance mechanisms. Upon completion of the initial Wiki writing process, every group is appointed to be responsible for an article of another group, in order to (i) improve it and (ii) write a summary. Log files show that students do not only read the article they are asked to improve, but also most other articles, possibly for comparison and inspiration purposes. A potential problem with this approach is that we frequently observe strong reservations against editing the work of others. Also, the imbalance of the workload poses a problem since some articles require more improvement than others.

The online-phase is followed by a final phase of attendance for two and a half days in class where additional 
games are played at a higher level of complexity. Whenever new aspects such as stock-keeping or licensing are introduced in a game, participants form teams of two students each managing a single firm to allow for reflection on and discussion of the new challenges. We also observed that information exchange typically does not only occur within each group during these games, but also between groups and thus among competitors. However, this willingness to cooperate tends to decrease as the ambition to win the game rises at a later stage. This holds true particularly for the final assessment games, where students can earn points that contribute (at a low percentage) to their grades. By including the gaming results into the grading scheme, we provide incentives that appear to be efficient in that they considerably enhance the involvement of students.

Reflection on actions and decisions plays a vital role in all management games. In addition to regular feedback during the game and in the discussions, participants therefore are required to individually reflect on their decisions and the whole management simulation at the end of the course by writing three seminar papers. In the first one, students report on their "ten most fatal" decisions and their consequences. Besides describing the circumstances and the mistake they made (e.g. failure to properly take inflation into account when determining prices), participants should also discuss possible strategies to avoid the respective problem. In order to foster understanding of the underlying model, students are required to produce a second seminar paper that describes basic elements of the management simulation and their interrelationships, both conceptually and formally. This serves as a starting point for team discussions about suggestions for extensions and improvements of the game, which result in a third seminar paper. Upon elaboration of this final paper, students concern themselves with methods of quantitative modeling. For most students, this phase serves as a "gentle" introduction to model building and simulation, which may be considered an additional benefit.

\section{TECHNICAL IMPLEMENTATION}

A computer-based simulation that can be used in the didactic design outlined in the previous section has to meet a number of key requirements. To begin with, the application has to support multiple usage modes in order to allow for various educational settings. For instance, during the first phase of the didactic process outlined in the previous section, which takes place in a classroom, the game is run locally on a single computer; the second phase is Internet-mediated, which implies that an intuitive user interface is required to allow participants to submit decisions and analyze results easily; the third phase is again conducted in a classroom setting where each participant uses a dedicated computer connected to a local area network. Moreover, participants cannot be expected to install additional software on their computers. They should rather be able to use any standard-compliant web browser on any platform to submit their decisions and access reports. Further to this, the application core itself should be platform-independent, as it is used both locally on Windows-based computers and on dedicated application servers running Linux. Moreover, it was decided that the implementation should rely on freely available software. Major design objectives were stability, resistance to manipulation, and extensibility of the system.
Next, a number of major functional requirements were identified. First, the application must provide an intuitive web-based user interface. Participants should be able to focus their attention entirely on the simulation rather than being distracted by usability issues. Second, the application should provide the instructor with extensive capabilities for model building in order to give her the freedom to create arbitrary scenarios. To facilitate the complex parameterization process, the system needs to provide appropriate mechanisms such as the ability to define smooth functions interactively in a graphical way. Third, it should be possible to tailor the simulation to the needs of both novice and experienced participants by limiting or extending the decision space during parameterization. Finally, the system must give ample and easily understandable feedback to provide a solid base for decisions and facilitate an effective learning process. Considering all functional and non-functional requirements, we chose Java Enterprise Edition [11] as an implementation platform and developed a distributed application consisting of four major components, as illustrated in Figure 4.

The central component that manages user accounts and game instances and executes the simulation model is hosted on an application server. It provides a web-based

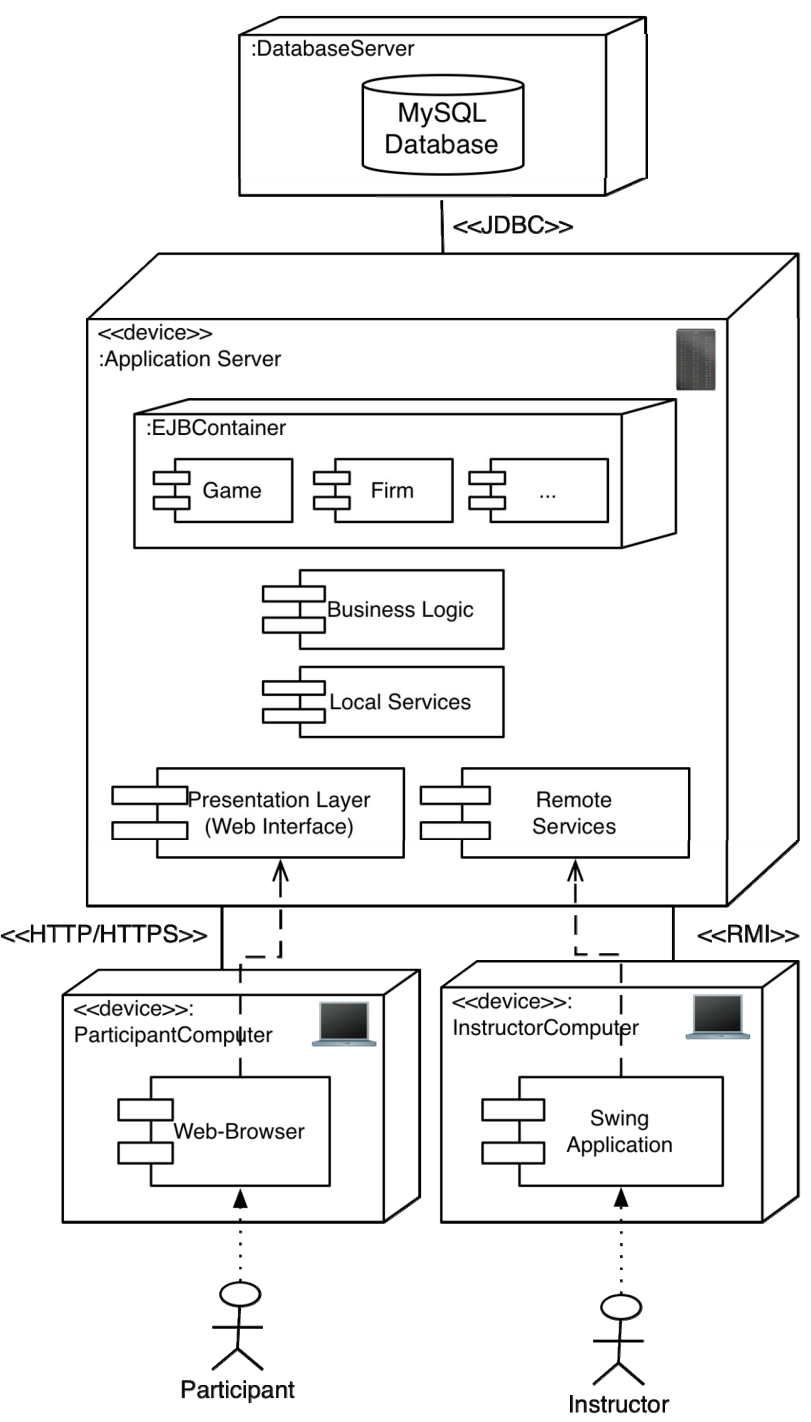

Figure 4. Deployment diagram. 


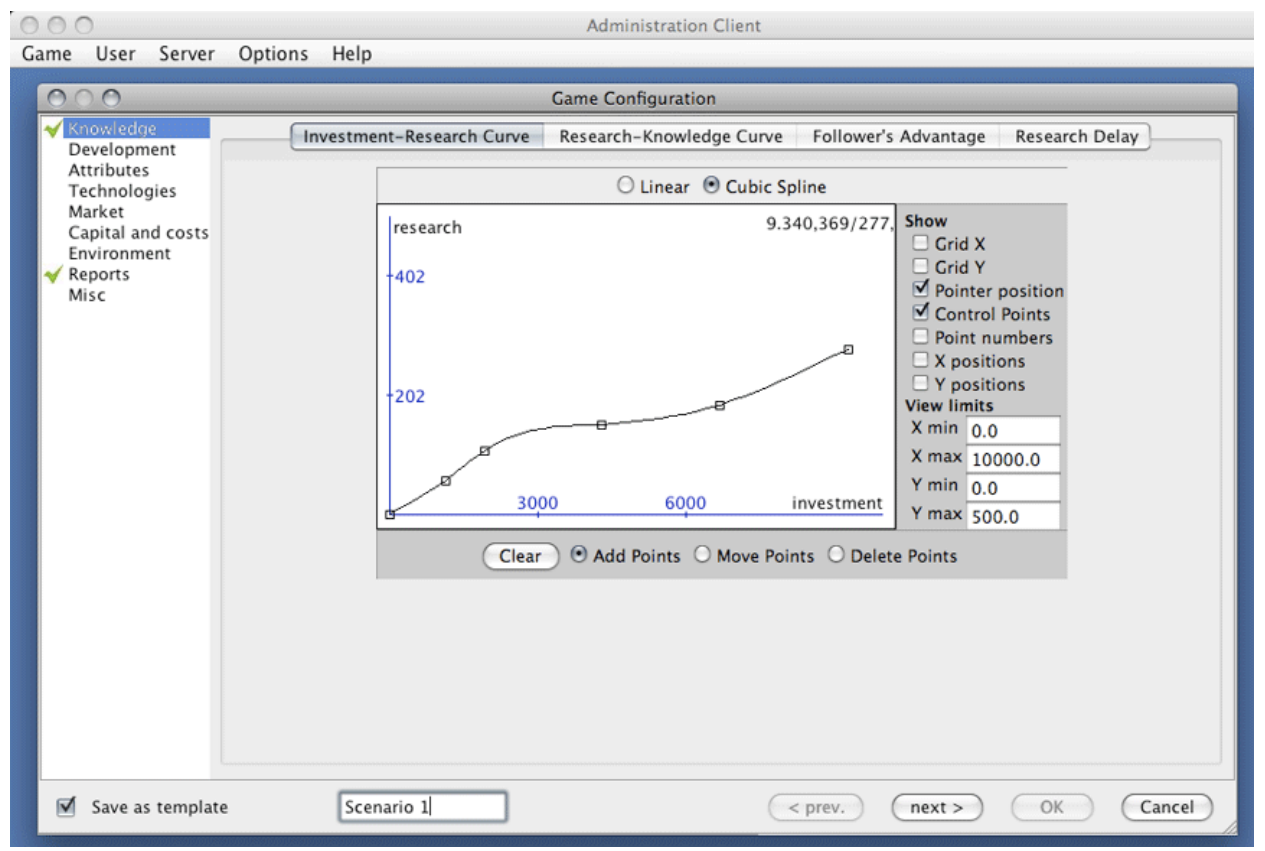

Figure 5. Scenario creation using the instructor tool.

interface for participants as well as remote services for an instructor client, which is used for the creation and parameterization of game instances, user account administration, assignment of participants to games, and entering decisions for participants in the second phase of the didactic process. The application server connects to a database server providing persistence.

Applying the concept of multi-tier architectures, graphical user interface, business logic, and persistence layers are strictly decoupled and the resulting services are connected through interfaces. This clear separation has significant benefits in terms of maintainability and extensibility, as it results in a modular system of independent components that can be exchanged or modified easily without affecting other parts of the system. Another key advantage of this approach is that it is relatively easy to connect multiple independent presentation layer components, such as a web interface for participants and an application client used by instructors. Finally, and possibly most importantly, a multi-tier approach is a good way to support the parameterization of management simulations for multiple educational settings, where for example the number of players, the scenario to use and the underlying simulation model may differ each time (cf. [12]).

The individual components depicted in Figure 4 can be distributed across several computers or run on a single machine. The core of the system is deployed on a JBoss J2EE application server, which is purely Java-based and can thus be run on a large number of operating systems. Container-managed persistence, a technique where the object-relational mapping is handled by the J2EE container the application runs in, is used to preserve the state of objects to a MySQL database. The web-interface relies on Java Server Faces, JSP, and Java Portlets. The instructor client is implemented in Java Swing and connects to the application server via remote method invocation (RMI). It provides a rich user interface, does not require installation, and is platform-independent. For an illustrative example of its use, see Figure 5.

\section{CONCLUSION}

The management game introduced in this paper complements more "traditional" instructional methods commonly used in technology management education in a blended-learning course design and provides an excellent opportunity for students to gain relevant first-hand experience through learning-by-doing. The service-oriented architecture of the application facilitates its use in various educational settings and its adaptation to the specific needs of participants. Learning success, however, is not solely determined by the quality of the underlying simulation model or its technical realization. These factors must rather be considered necessary conditions. When it comes to achieving learning outcomes, the integration in a broader didactic design plays a key role for success. For this reason we suggest combining the game-based learning approach of the management simulation with various additional elements into a didactic design that encompasses (i) continuous peer-to-peer knowledge exchange, both onand offline, (ii) teamwork and team play, (iii) written expost reflection on decisions and discovered relationships, (iv) suggestion of extensions to the simulation in a seminar paper, which encourages participants to gain a deeper understanding of the underlying model and may serve as an introduction to mathematical modeling and (v) theoretical considerations regarding various topics related to the simulation, which are prepared by participants in the form of Wiki articles. In order to assure quality of the contributions and encourage the dissemination of information among participants, these articles are subject to obligatory peer review.

Still, our management game may be complemented in several directions. First, the integration of online communication directly into the management game would eliminate the media gap caused by the use of various external communication channels and, thus, help to alleviate the lack of rich communication and information exchange during the online game. Learning blogs could be used to 
allow the participants to record and analyze their thoughts, strategies and decision-making processes during the game. Next, additional game options like multiple (international) markets or cross-licensing of patent portfolios with balancing royalty payments and/or the introduction of computer-controlled rivaling firms would allow for new possibilities.

\section{REFERENCES}

[1] S. Liyanage and P. S. Poon, "Technology and innovation management learning in the knowledge economy: a techno-managerial approach," Journal of Management Development, vol. 22, no. 7, pp. 579-602, 2003. doi:10.1108/02621710310484740

[2] "Master of Engineering Management Programs Consortium Mission," http://www.mempc.org. Accessed 01.11.2009.

[3] L. K. Langford, "Surf's up: transforming engineering education," IEEE Engineering Management Review, vol. 37, no. 1, pp. 91-92, 2009. doi:10.1109/EMR.2008.2012090

[4] D. N. Mallickand and A. Chaudhury, "Technology management education in MBA programs: a comparative study of knowledge and skill requirements," Journal of Engineering and Technology Management, vol. 17, no. 2, pp. 153-173, 2000. doi:10.1016/S0923-4748(00)00019-9

[5] D. C. Lane, "A resurgence of management simulations and games," Journal of the Operational Research Society, vol. 46, no. 5, pp. 604-625, 1995.

[6] A. J. Faria, "Business simulation games: current usage levels: a ten year update," Simulation \& Gaming, vol. 29, no. 3, pp. 295308, 1998. doi:10.1177/1046878198293002

[7] A. J. Faria and W. J. Wellington, "A survey of simulation game users, former-users, and never-users," Simulation \& Gaming, vol. 35, no. 2, pp. 178-207, 2004. doi:10.1177/1046878104263543

[8] A. J. Faria, D. Hutchinson, W. J. Wellington, and S. Gold, "Developments in business gaming: a review of the past 40 years," Simulation Gaming, vol. 40, no. 4, pp. 464-487, 2009. doi: $10.1177 / 1046878108327585$
[9] J. W. Gentry, "What is experiential learning?," in Guide to business gaming and experiential learning, J. W. Gentry, Ed. Brunswick NJ: Nichols/GP Publishing, 1990, pp. 9-20.

[10] J. M. Hughes, "The body of knowledge in management education," Management Education and Development, vol. 19, no. 4, pp. 301-310, 1988.

[11] Sun Microsystems, Java 2 Platform Enterprise Edition Specification, v1.4. CA, USA, 2003.

[12] S.-P. van Houten and A. Verbraeck, "Supporting parametrization of business games for multiple educational settings," in Proc. 2007 Winter Simulation Conference, Washington D.C., 2007, pp. 23222326. doi:10.1109/WSC.2007.4419871

\section{AUTHORS}

M. Günther is Junior-Professor of Technology Management and Entrepreneurship at the Faculty of Business Administration and Economics, Bielefeld University, Universitaetsstr. 25, 33615 Bielefeld, Germany (e-mail: markus.guenther@uni-bielefeld.de).

E. Kiesling is junior researcher and lecturer at the Faculty of Business, Economics, and Statistics, University of Vienna, Bruenner Str. 72, 1210 Vienna, Austria (e-mail: elmar.kiesling@univie.ac.at).

C. Stummer is Professor of Innovation and Technology Management at the Faculty of Business Administration and Economics, Bielefeld University, Universitaetsstr. 25, 33615 Bielefeld, Germany (e-mail: christian.stummer@uni-bielefeld.de).

A version of this paper was presented at the IEEE EDUCON 2010 conference and selected for submission to this journal. It received an award as Most Innovative Paper Regarding Engineering Education in this conference.

Submitted, October, 08, 2010. Published as resubmitted by the authors on March $1^{\text {st }}, 2011$. 